\title{
The Physics \& Design Issues of a High Gain FEL
}

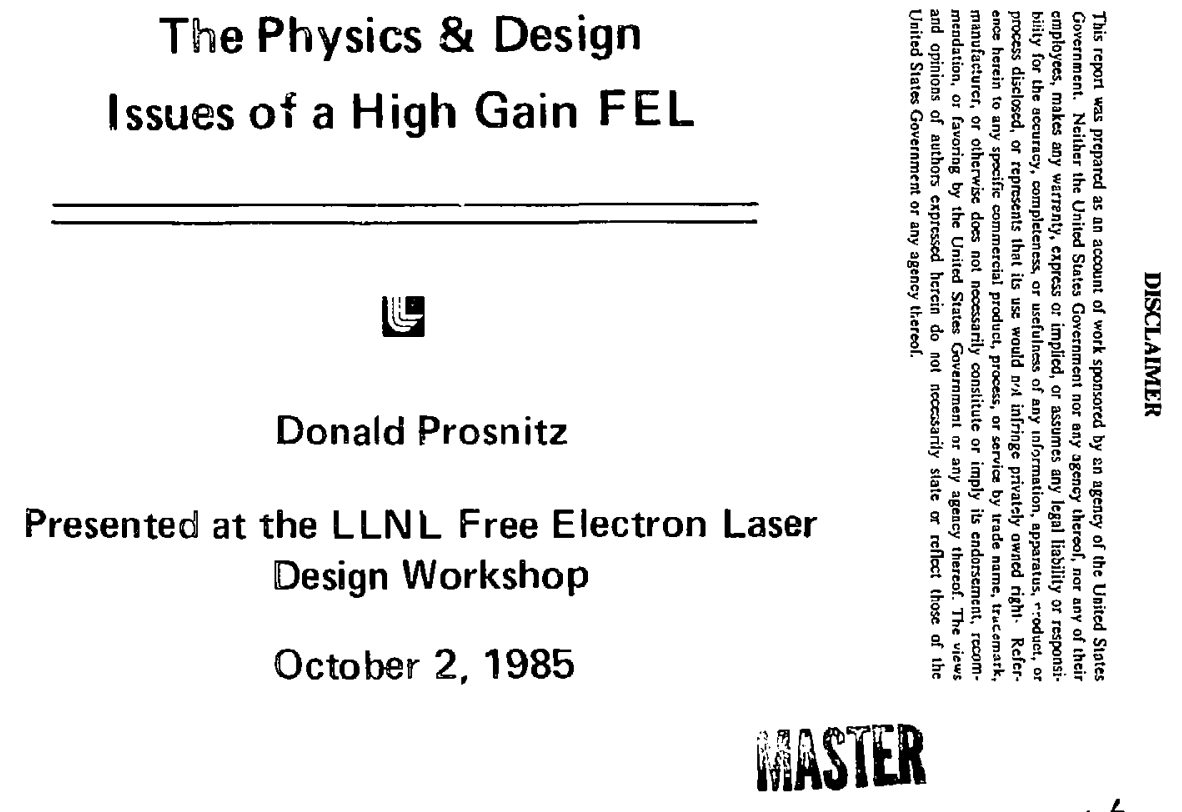
VAASTER 


\section{The free-electron laser}

- Directly converts the kinetic energy of a relativistic electron beam into light

- Has no solids, liquids, or gases that can ionize or be damaged inside the leser

- Can be very efficient 
- The coupling between electrons and light

- The wiggler and wiggle motion

- The laser electric field

$-\vec{v} \cdot \vec{E}$ coupling

- Resonance

- Electron beam properties that affect the FEL

- Emittance

- Energy spread

- Energy loss

- Tepering the wiggler 


\section{Overview (cont)}

- Focusing the electron beam

- Guiding the light by the electron beam

- Scaling

[ 0 High-gain, single-pass FEL amplifiers only will be discussed here-not FEL oscillators or RF linac driven FEL's] 


\section{ENERGY TRANSFER IN THE FREE ELECTRON LASER (FEL)}

í

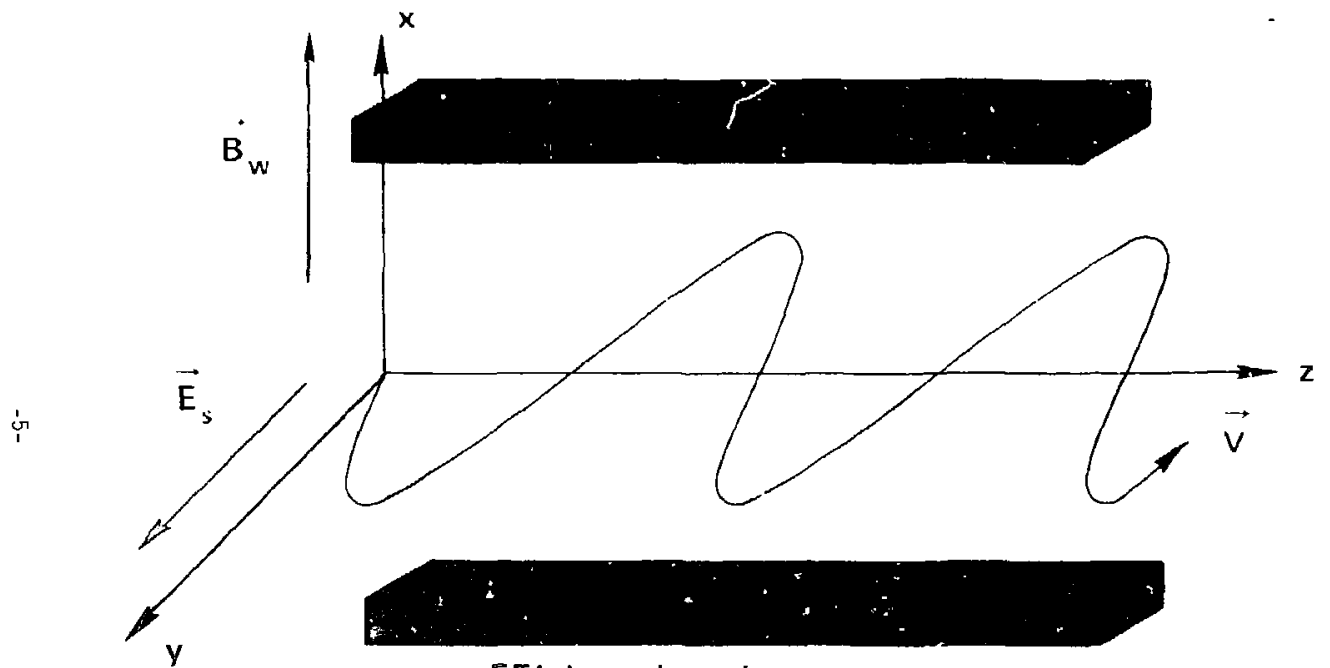

FEL intensity gain:

$\frac{d l_{s}}{d Z} \propto$ [Electron energy loss] $\propto \vec{V} \cdot \vec{E}_{s}$

04-13-0680-1866 
- Horizontally polarized:

$$
\begin{aligned}
& -E_{x}=E_{s} \cos (k z-\omega t+\phi) \\
& - \text { with } k=2 \pi / \lambda, \omega=2 \pi \nu
\end{aligned}
$$

ELF: $\lambda=8.6 \mathrm{~mm}(34.6 \mathrm{GHz})$

PALADIN: $\lambda=10.6 \mu \mathrm{m}=10,600 \AA$

- $E_{s}=E_{s}(x, y), \phi=\phi(x, y)=$ phase 


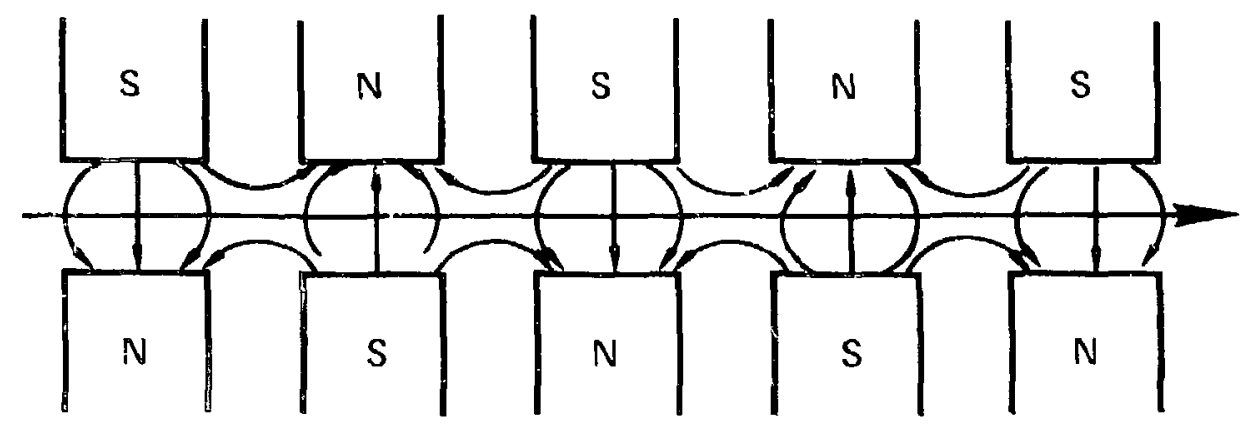

- Electron beam

- Magnetic fieló linos

On midplane $\vec{B}=B_{y 0} \cos k_{w} z \quad k_{w}=2 \pi / \lambda_{w}$ 


\section{The wiggler - examples}

- ELF - the microwave FEL next door

$$
\begin{aligned}
& \lambda_{w}=9.8 \mathrm{~cm} \\
& B_{y o} \cong 3.8 \mathrm{kG}(0-5 \mathrm{kG}) \\
& \sim 3 \mathrm{~cm} \text { gap between magnets } \\
& L_{w}=3 \mathrm{~m} .
\end{aligned}
$$

- PALADIN - the infrared FEL to be built at ATA

$$
\begin{aligned}
& \lambda_{w}=8 \mathrm{~cm} \\
& B_{\text {yo }} \cong 2.5 \mathrm{kG} \\
& \sim 3 \mathrm{~cm} \mathrm{gap} \\
& L_{w} \cong 25 \mathrm{~m} . \\
& \quad 2 \mathrm{~cm} \leqq \lambda_{w} \leqq 10 \mathrm{~cm} \\
& 1 \mathrm{kG} \leqq \mathrm{B}_{\mathrm{yo}} \leqq 5 \mathrm{kG} \\
& 0.5 \mathrm{~m} \leqq \mathrm{~L}_{\mathrm{w}} \leqq 2 \mathrm{~m} .
\end{aligned}
$$




\section{Wiggle motion - examples}

$$
\begin{array}{ll}
\left|\frac{v_{x}}{c}\right|_{\max } \cong \frac{e B_{y o}}{\gamma \text { mck }_{w}}, & \begin{array}{l}
\text { an angle at which } \\
\text { electrons cross the } \\
x=0 \text { plane }
\end{array} \\
|x|_{\text {max }} \cong \frac{e B_{y o}}{\gamma m c k_{w}^{2}}, & \begin{array}{l}
\text { maximum displacement } \\
\text { from straight-line orbits }
\end{array}
\end{array}
$$

\begin{tabular}{l|cc}
\multicolumn{1}{c}{} & ELF & PALADIN \\
\cline { 2 - 3 }$\gamma$ & 7 & 100 \\
Angle & $0.5 \mathrm{rad}$ & $0.02 \mathrm{rad}$ \\
Displacement & $8 \mathrm{~mm}$ & $200 \mu \mathrm{m}=0.2 \mathrm{~mm}$
\end{tabular}




\section{Eiectron-laser coupling}

- Moving with the average motion of an electron

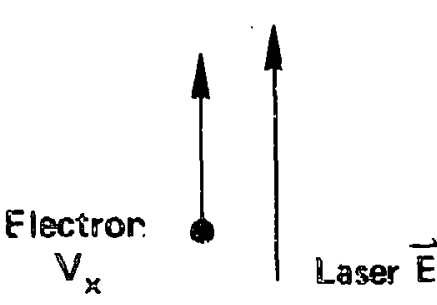

Time $t_{0}$

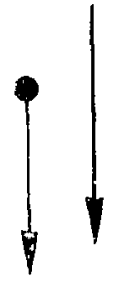

$$
t_{0}+\frac{\lambda_{w}}{2 V_{z}}
$$

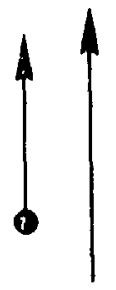

$$
t_{0}+\frac{\lambda_{w}}{v_{z}}
$$

$\circ \vec{V} \cdot \vec{E}$ albusys greater than zero $\Rightarrow$ steady energy loss of olectron $\Rightarrow$ steady energy gain of laser 


$$
\begin{aligned}
& \frac{d \gamma}{d t}=-\frac{e}{m c^{2}} \quad \vec{v} \cdot \vec{E}=-\frac{e}{m c^{2}} \quad v_{x} E_{i i} \\
& =-\frac{e}{m c^{2}} \frac{e B_{y o}}{\gamma m k_{w}} E_{s} \sin k_{w} z \cos (k z-\omega t+\phi) \\
& \propto-\sin \left[\left(k+k_{w}\right) z-\omega t+\phi\right] \\
& +\sin \left[\left(k-k_{w}\right) z-\omega t+\phi\right]
\end{aligned}
$$

- If $\left.i k+k_{w}\right) z-\omega t+\phi>0$ and $\sim$ constant, then

$$
\frac{d \gamma}{d t}<0
$$


If, for a single $e^{-},\left(k+k_{w}\right) z-\omega t+\phi \cong$ const then

- $\left(k-k_{w}\right) z-\omega t+\phi \cong-2 k_{w} z+$ const $\Rightarrow$ rapidly varying and zero average for second term

- $\underbrace{\frac{\mathrm{dz}}{\mathrm{dt}} \cong \frac{\omega}{\mathrm{k}+\mathrm{k}_{\mathrm{w}}}}<\mathrm{c}$, since $\frac{\omega}{\mathrm{k}}=\lambda \nu=\mathrm{c}$ the $F E L$ resonance condition 
- $\psi \equiv\left(k+k_{w}\right) z-w t+\phi$

- Slowly varying phase in a potential well of width $\cong \lambda$

- $\frac{d \gamma_{i}}{d t} \propto-\sin \psi_{i}$ for $i^{\text {th }}$ electron

- $\psi_{i}>0$ means wiggle motion in phase with $\vec{E}$ of laser $\Rightarrow$ energy loss

- $\psi_{i}<0$ means wiggle motion out of phase with $\vec{E}$ of laser $\Rightarrow$ energy gain

- Sign of $\psi_{i}$ is random for initial $\mathrm{e}^{-}$beam 
The Free Electron Laser is tuned by changing the electron beam's parallel energy

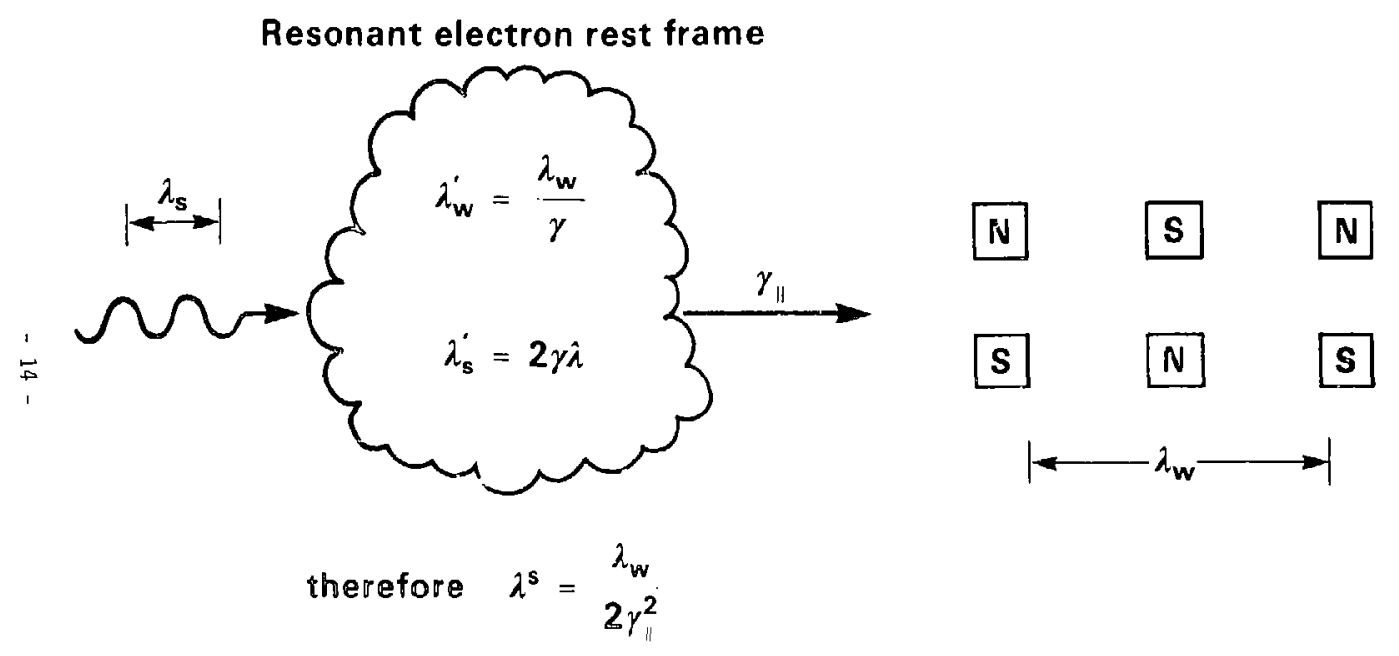




\section{Gain mechanism}

- Electrons with $\psi_{\mathrm{i}}<0$ gain energy, move faster, move toward $\psi_{i} \cong 0$

- Electrons with $\psi_{i}>0$ lose energy, slow down, move toward $\psi_{i} \cong 0$

$\Rightarrow$ Spontaneous bunching at $\psi_{\mathrm{i}} \cong 0$, where electrons neither gain nor lose energy,

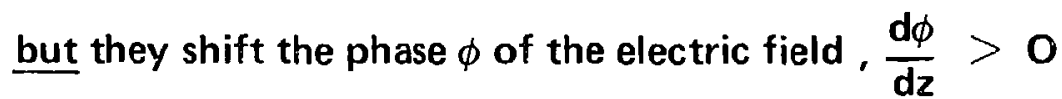


- Emittance - a measure of the "temperature" of the transverse $(x, y)$ electron velocities $\Rightarrow$ electrons with same $\gamma$ but different random $v_{x}, v_{y}$ (superimposed on iviggle motion) will have different $\beta_{\|}$'s scattered around resonance

5

- Energy spread 


\section{Electron beam emittance characterizes}

the electron's average trajectories

$\underline{\text { Trajectories }}$

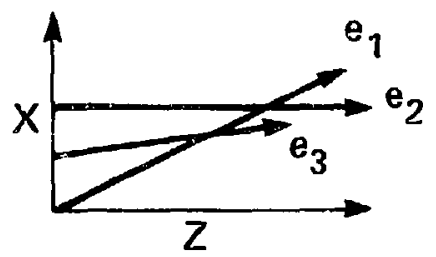

Phase space

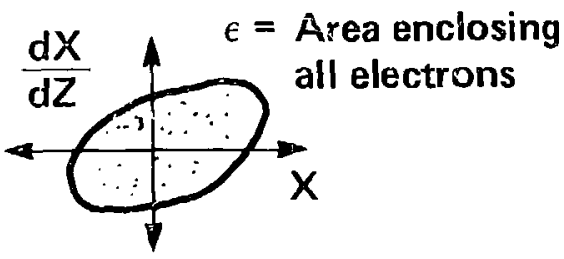

- The shape in phase space may change, but for constant energy, the area is preserved

- For accelerated or deceierated beams the normalized emittance, $\gamma E$, is constant

- The swread in parallel energy $\left(\Delta \gamma_{\|}\right)$can be related to the phase space area

- $g=\frac{2 l \pi^{2}}{\gamma^{2} d^{4} V} \approx \frac{1 \pi^{2}}{(\gamma \epsilon)^{2}}=$ beam brightness 


\section{$\mathrm{e}^{-}$beam properties (2)}

- Energy loss - essential to gain mechanism and FEL operation

- As $\mathrm{e}^{-}$lose energy, they drop sut of resonance, or (more precisely) they fall back to $\psi<0$ and start taking energy back from trie light Hence TAPERING 


\section{Tapering the wiggler}

$$
\lambda=\frac{\lambda_{w}}{2 \gamma_{\|}{ }^{2}}=\lambda_{w} \frac{\left(1+a_{w}^{2}\right)}{2 \gamma^{2}} \quad \begin{array}{r}
\text { resonance } \\
\text { condition }
\end{array}
$$

$\lambda$ fixed, laser wavelength

As $\gamma$ decreases (energy loss), resonance can be maintained if

- $\lambda_{w}$ decreases down the wiggler, or

- $a_{w} \propto B_{y o}$ derreases down the wiggler, or

- some combination

LLNL approach is to decrease $B_{y o}$ with increasing $z$ - experimental flexibility 


\section{Taper design (in theol y)}

- Set $a_{w}(z)=\frac{e}{\sqrt{2 \text { ckk }_{w}}} B_{y o}(z)$ so that one electron stays at consiant $\psi=\psi_{r}$ as it decelerates

- As $a_{w}$ and $\gamma$ both decrease, $\gamma_{\|}$(and $\beta_{\|}$) remain constant for for this design electron

- All other $\mathrm{e}^{-}$decelerate differentiy (or not at all!) 


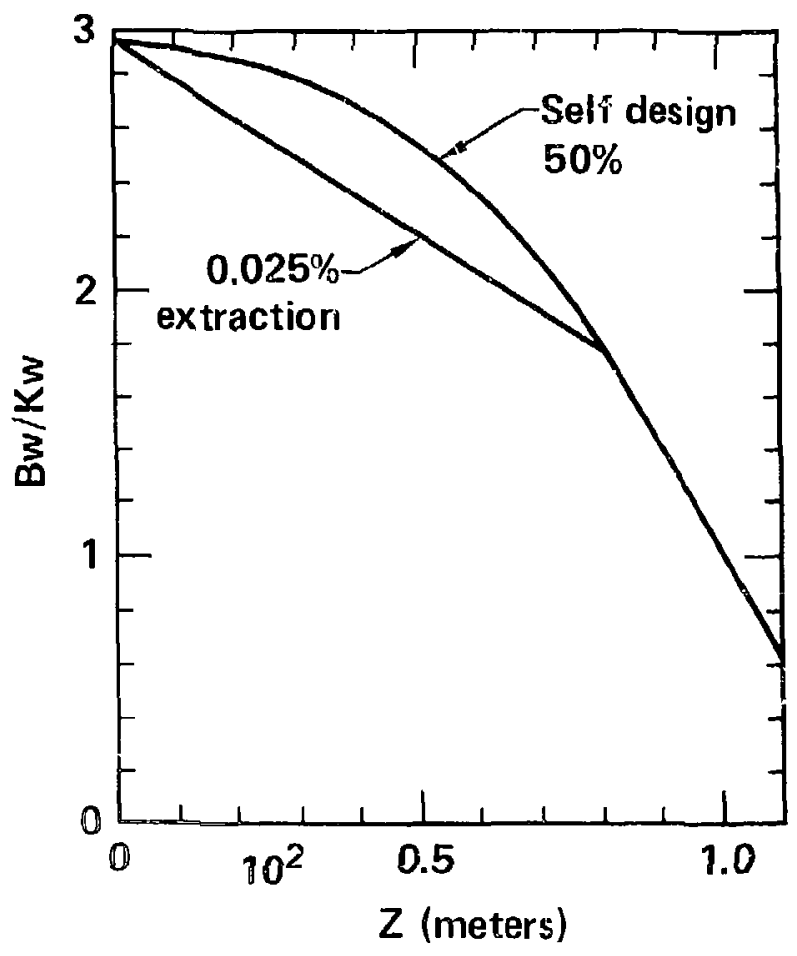




\section{Wiggler field}

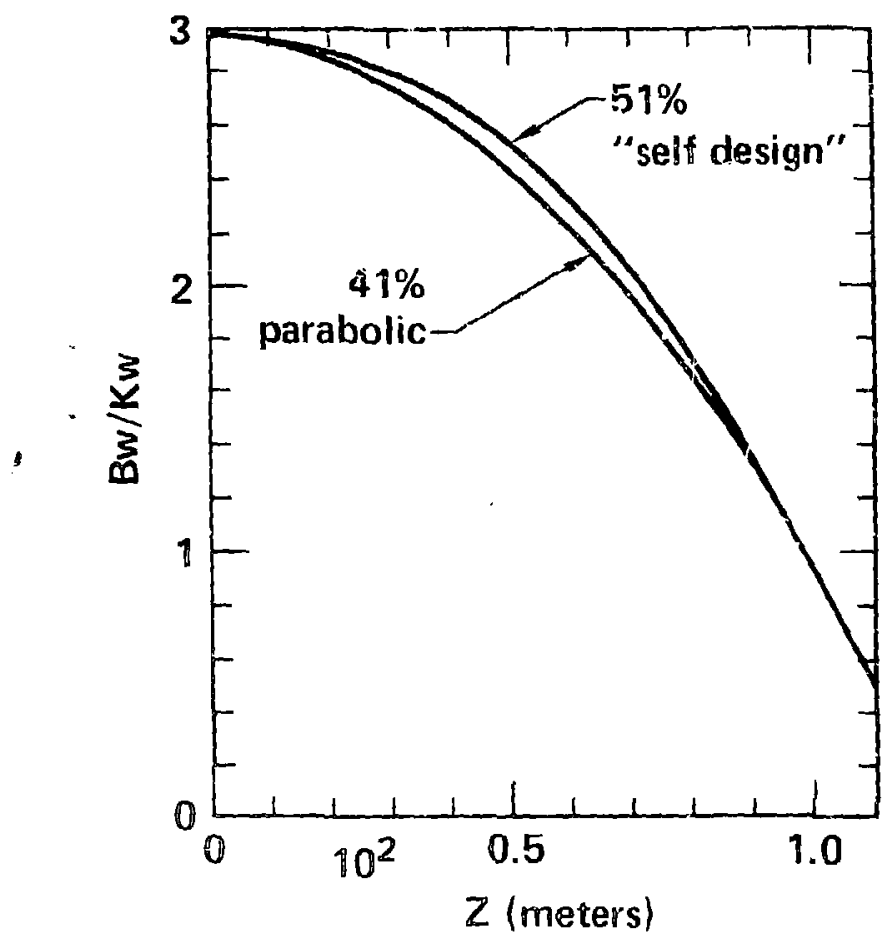




\section{Trapped electrons}

- Elecirons near the design electron that stay within $-\pi<\psi<\pi$, and decelerate with the design electron are "trapped" in the potential vuell

- Trapped electrons undergo "synchrotron" oscillations around the design electron
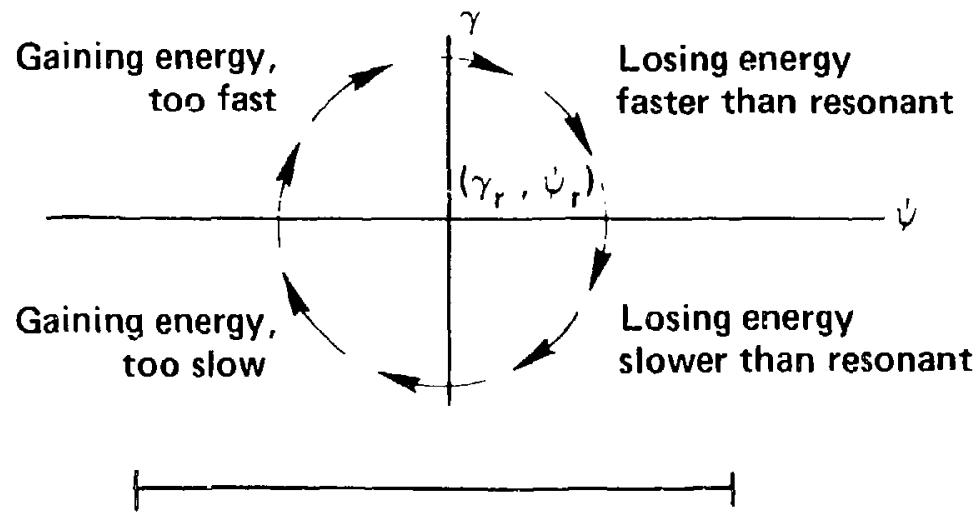

One optical wavelength 


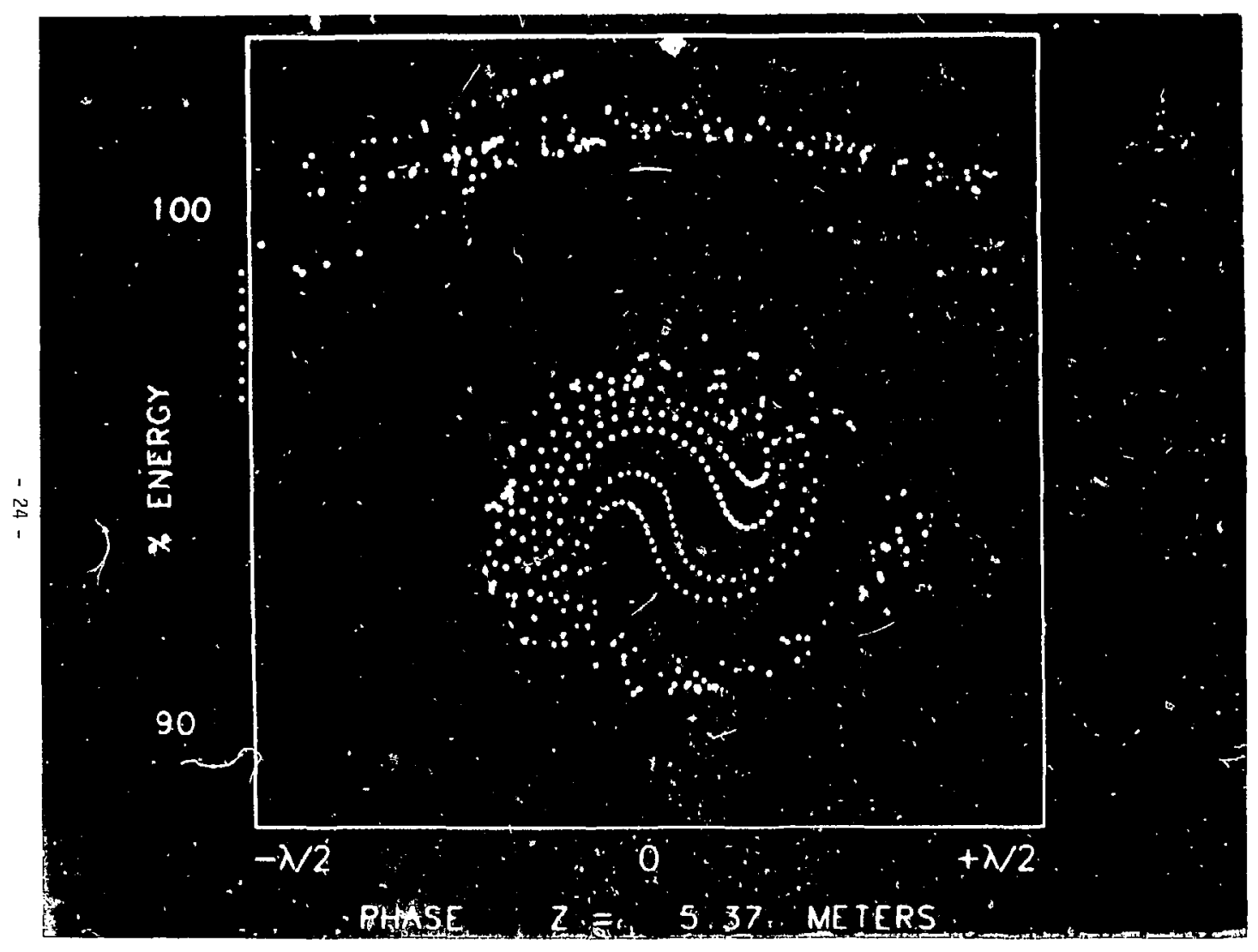




\title{
Trapped electrons (2)
}

- Oniy trapped electrons are continuously decelerated in a tapered wiggler - so

\author{
Power extracted from $\mathrm{e}^{-}$beam $\cong$ \\ Initial $e^{-}$beam power $X$ \\ Trapped fraction $X$ \\ Fractioinal deceleration in $\gamma$
}

- Simplest design tradeoff - too rapid deceleration $\Rightarrow$ less trapping 


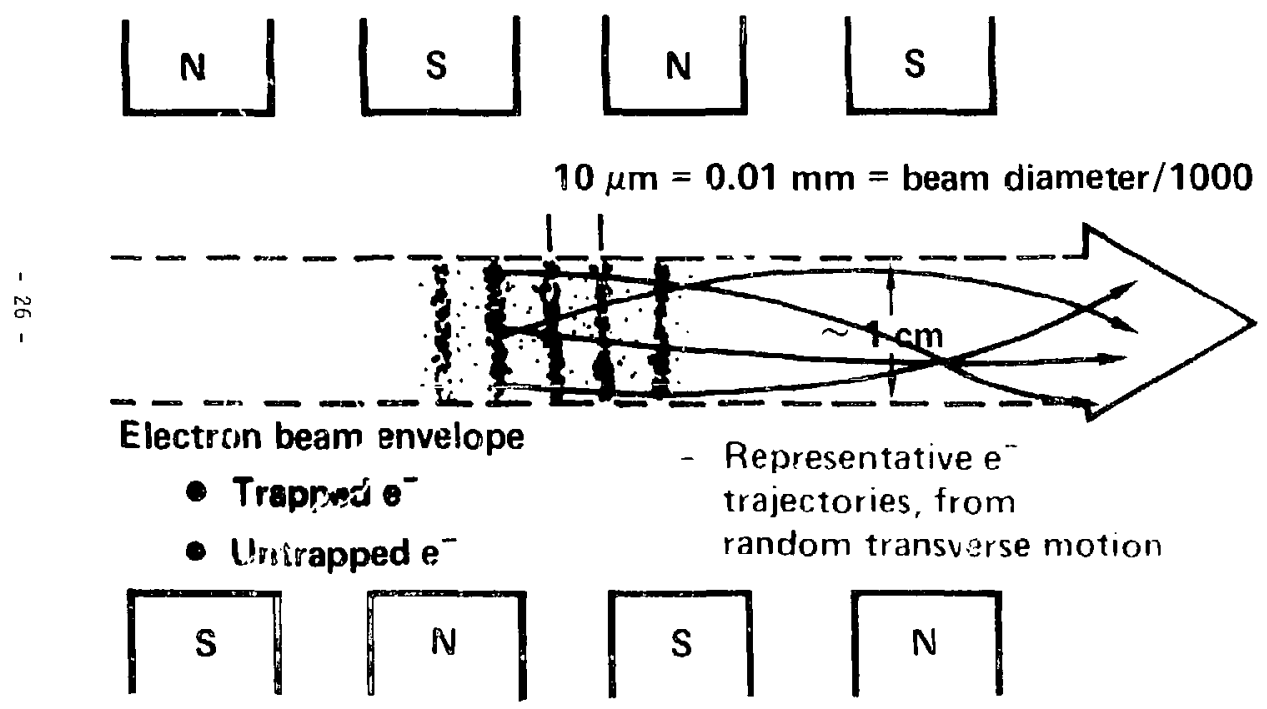




\section{Transport of an optically bunched beam (2)}

- Electron beam focusing must preserve $10 \mu$ bunches inside $1 \mathrm{~cm} \mathrm{e}^{-}$beam

- Focusing with curved pole faces can (in theory) accomplish this feat 


\section{External focusing in linear wigglers can lead to extensive detrapping}

- Our tapered wiggler amplifier designs lower "buckets" of trapped particles many bucket heights over the full wiggler length

$\rightarrow$ Detrapping can strongly reduce gain

- Long linear wigglers require focusing in the wiggle plane

- External focusing (e.g. quadrupoies) is usual choice $\rightarrow\left(a_{w}^{2}+\gamma^{2} \beta_{\perp}^{2}\right)$ varies over an individual particle's betatron orbit

$\rightarrow \gamma_{\|}$variation destroying FEL resonance $\rightarrow$ detrapping

- In worst case, resonance between betatron and synchrotron motion can lead to resonant detrapping exceeding that from curved optical phase fronts

- Simulation runs confirmed problems with quadrupole focusing 


\section{Curved pole faces provide focusing solution}

- Curved, parabolically-shaped pole faces give focusing in both planes

- Invariance of $\left(a_{w}^{2}+\gamma^{2} \beta_{\perp}^{2}\right)$ retained over a betatron orbit

- Focusing in wiggle plane $(x)$ due to non-zero average of $\mathbf{B}_{y}$ over a wiggle period

- Betatron frequencies in wiggle and non-wiggle planes are given by

$$
\begin{aligned}
& k_{\beta, y}=k_{y} a_{w} / \gamma, k_{\beta, x}=k_{x} a_{w} / \gamma \text { where } \\
& k_{x}^{2}+k_{y}^{2}=k_{w}^{2}
\end{aligned}
$$

- If $k_{x}=k_{y}=k_{w} / \sqrt{ } 2$

$\rightarrow$ an injected axisymmetric beam remains so through the wiggler

$\rightarrow$ linear wiggler performs identically to helical wiggler of same rms magnetic field strength except for Bessel function coupling factor $(=0.9)$ between particles and field 


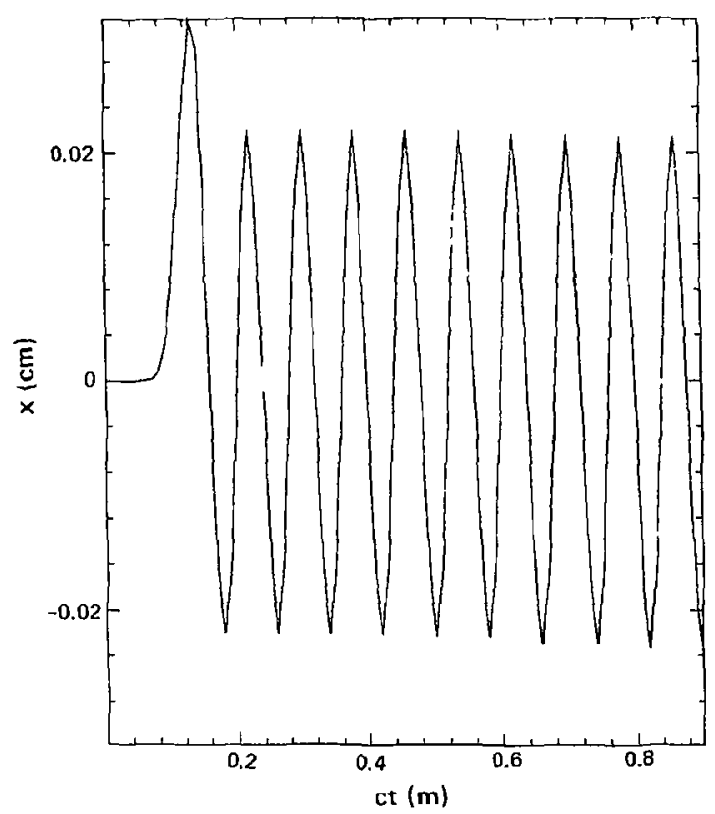


- Diffraction - the laser light would, in vacuum, spread out

$$
\begin{aligned}
& E_{s}(r) \propto e^{-r^{2} / w^{2}} \\
& \left.\begin{array}{c}
\text { laser beam } \\
\text { radius at } z
\end{array}\right|_{\begin{array}{c}
\text { smallest beam } \\
\text { radius ("waist") }
\end{array}} ^{w_{0}\left[1+\left(\frac{z}{z_{R}}\right)^{2}\right]} \cong w_{0} z / z_{R} \\
& z_{R} \equiv \frac{\pi w_{0}^{2}}{\lambda} \text { is "Rayleigh range" } \\
& =\text { characteristic expansion distance }
\end{aligned}
$$




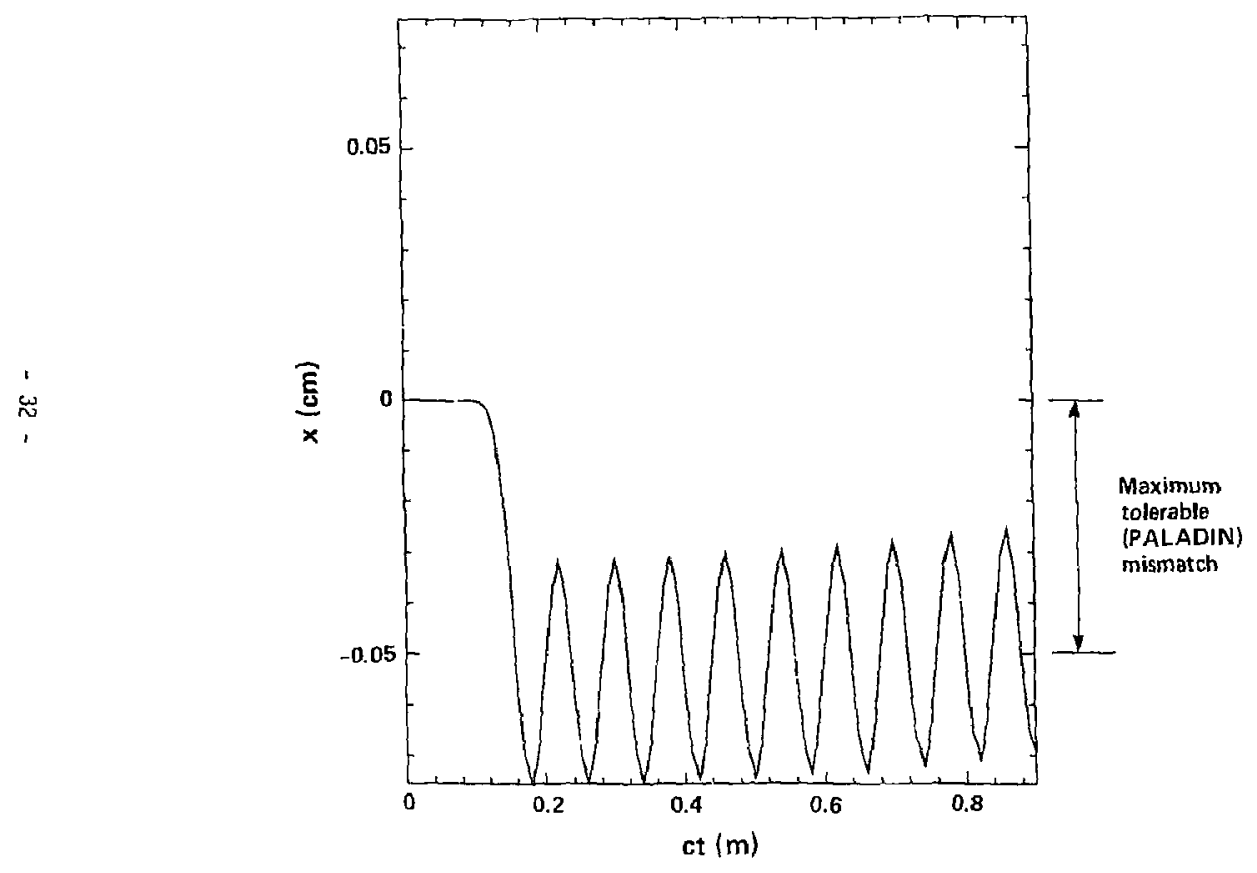




\section{Mismatched electron orbit}

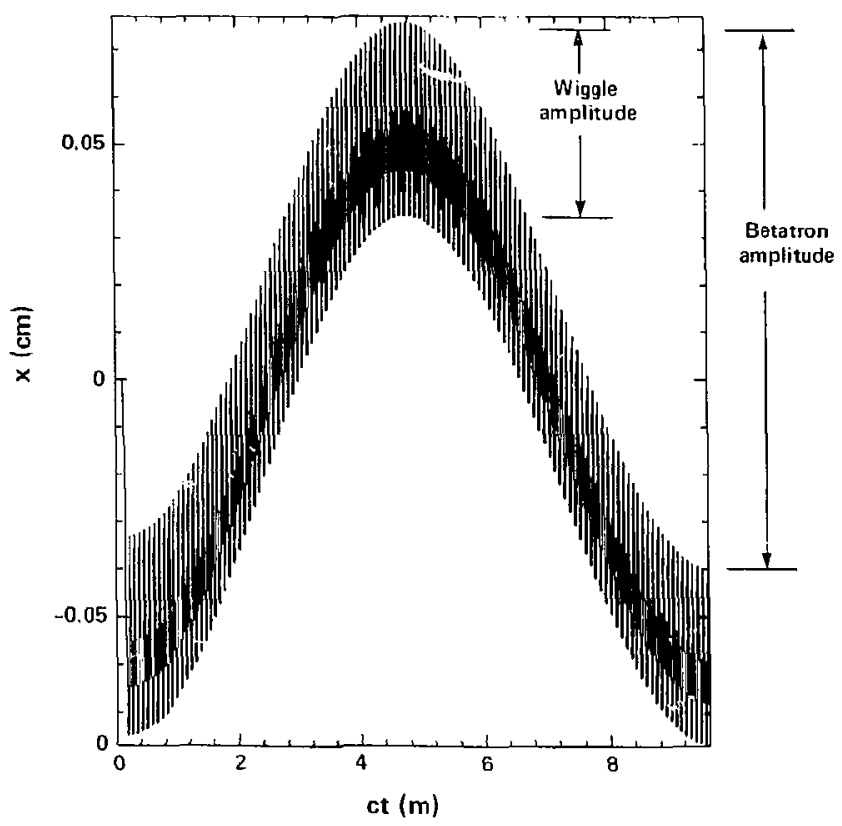




\section{Diffraction}

- For long wigglers, diffraction could be a problem: the laser light can diffract away from the electron beam, reducing (or eliminating) the electronlaser coupling

But

- Optically bunched electron beam has an effective index of refraction $n>1$, and can guide the light like an optical fiber 
- $n>1$ means $d \phi / d z>0$; ie., phase velocity of light reduced

- Optical fiber acts as a distributed lens, trying to focus the light toward the axis

- Light propagates with constant profile along a fiber, as the focusing precisely balances diffraction

- Electron bunches with $\psi \cong \mathbf{0}$ produce no gain, but phase shift $\mathrm{d} \phi / \mathrm{dz}>0 \Rightarrow n>1$ 
A bunched electron beam behaves as an optical fiber and guides the optical beam in a high gain FEL
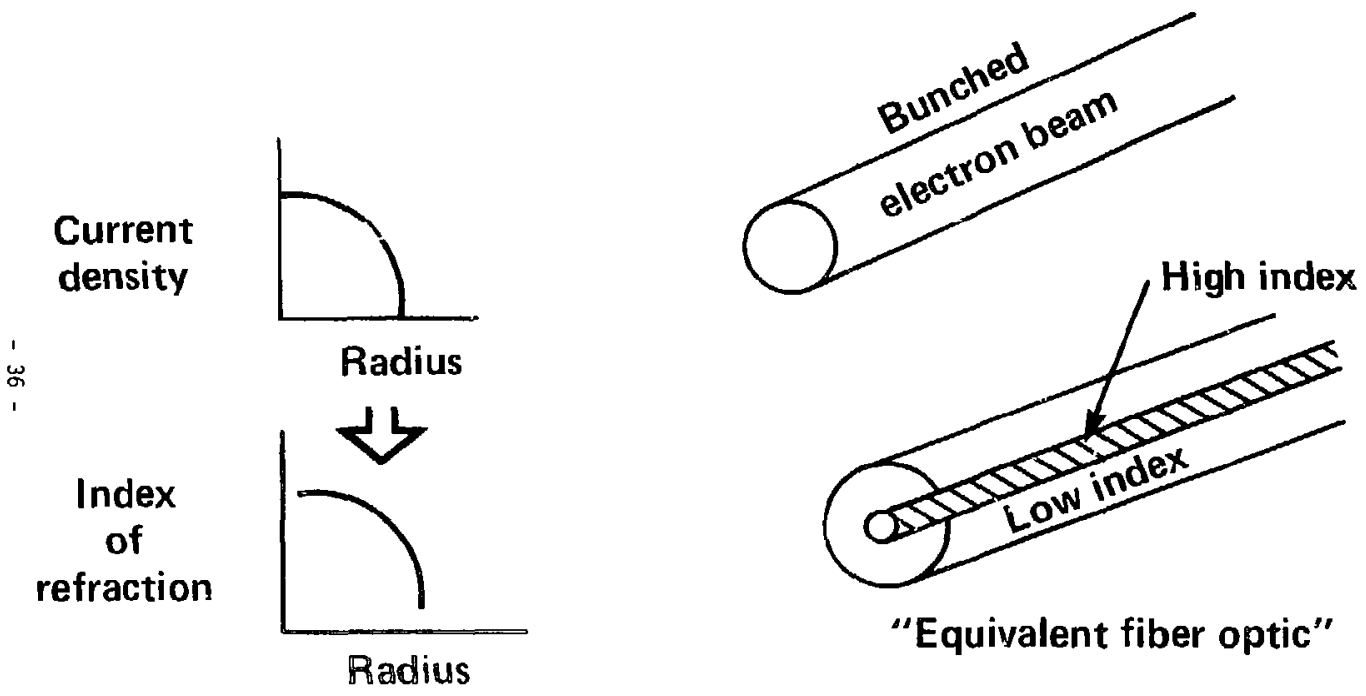
Why do we need high energy?

$$
\begin{aligned}
& \text { Relation } \\
& \lambda_{s}=\frac{\lambda_{w}}{2 \gamma^{2}}\left(1+a_{w}^{2}\right) \\
& \text { Consequence } \\
& \text { High } \gamma, \text { small } \lambda_{w} \\
& \$ / \mathrm{MeV} \\
& \underset{v}{\omega} \frac{d \gamma}{d z}=\frac{e E_{s}}{\sqrt{2} m c^{2}} \frac{a_{w}}{\gamma} \sin \psi \\
& \underset{\text { small } \gamma}{\text { Large }} \mathrm{E}_{\mathrm{s}} \text {, large } \mathrm{a}_{\mathrm{w}} \text {, } \\
& \text { Long wigglers } \\
& \text { may be diffraction } \\
& \text { limited }
\end{aligned}
$$

- Small $\lambda_{w}$ limits maximum acceptable emittance and hence current

o Small $\lambda_{w}$ limits maximum $a_{w}$ 
A shorter wiggler period requires a reduced electron beam emittance

$$
\frac{\Delta \gamma_{\|}}{\gamma_{\|}} \approx \frac{\left(2 \pi a_{w}\right)^{2}}{2} \frac{1}{1+a_{w}^{2}}\left(\frac{r_{b}}{\lambda_{w}}\right)^{2} F\left(d^{4} V\right)
$$

- $r_{b} / \lambda_{w}$ should be held constant

$$
\left(\frac{r_{b}}{\lambda_{w}}\right)^{2}=\frac{1}{2 \pi a_{w}}\left(\frac{\gamma \bar{c}}{\lambda_{w}}\right)
$$

- Beam emittance musc be reduced linearly with $\lambda_{w}$

- Beam current is consequently reduced as $\lambda_{w}^{2}$

(fixed brightness) 
A shorter wiggler period reduces the maximum $a_{w}$

$$
\begin{aligned}
& -\mathrm{a}_{\mathrm{w}}(\mathrm{rms}) \\
& \mathrm{g}=\text { full magnet gap } \approx \lambda_{\mathrm{w}} / 3
\end{aligned}
$$

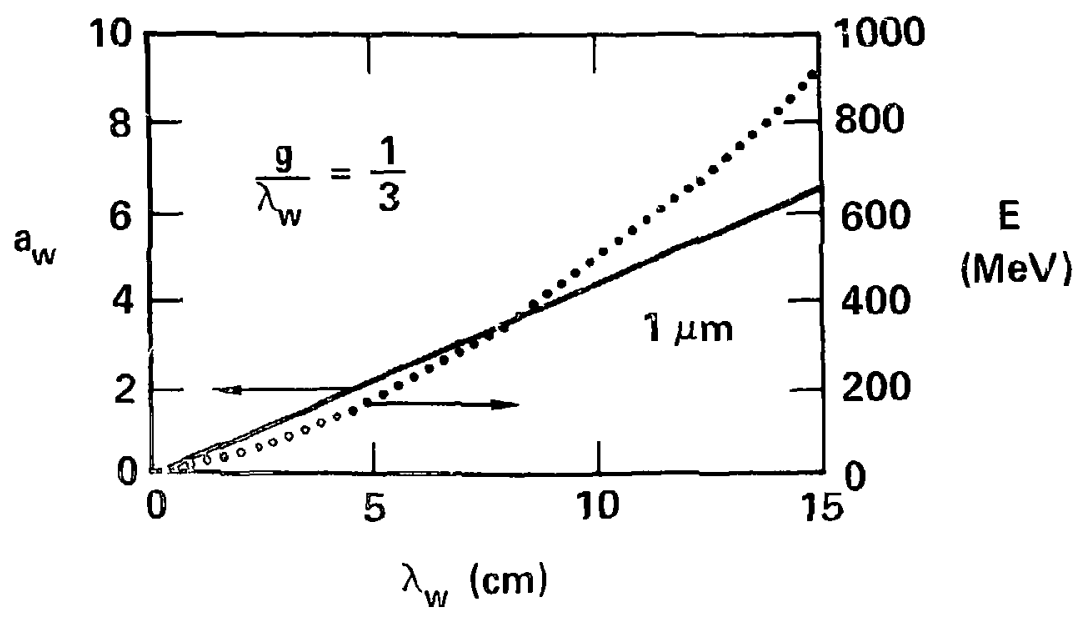


There are several pathways to a .5 micron FEL

Constant gain, 0 is $\mathbf{1 0 . 6}$ microns, ${ }^{*}$ is .5 microns

iे

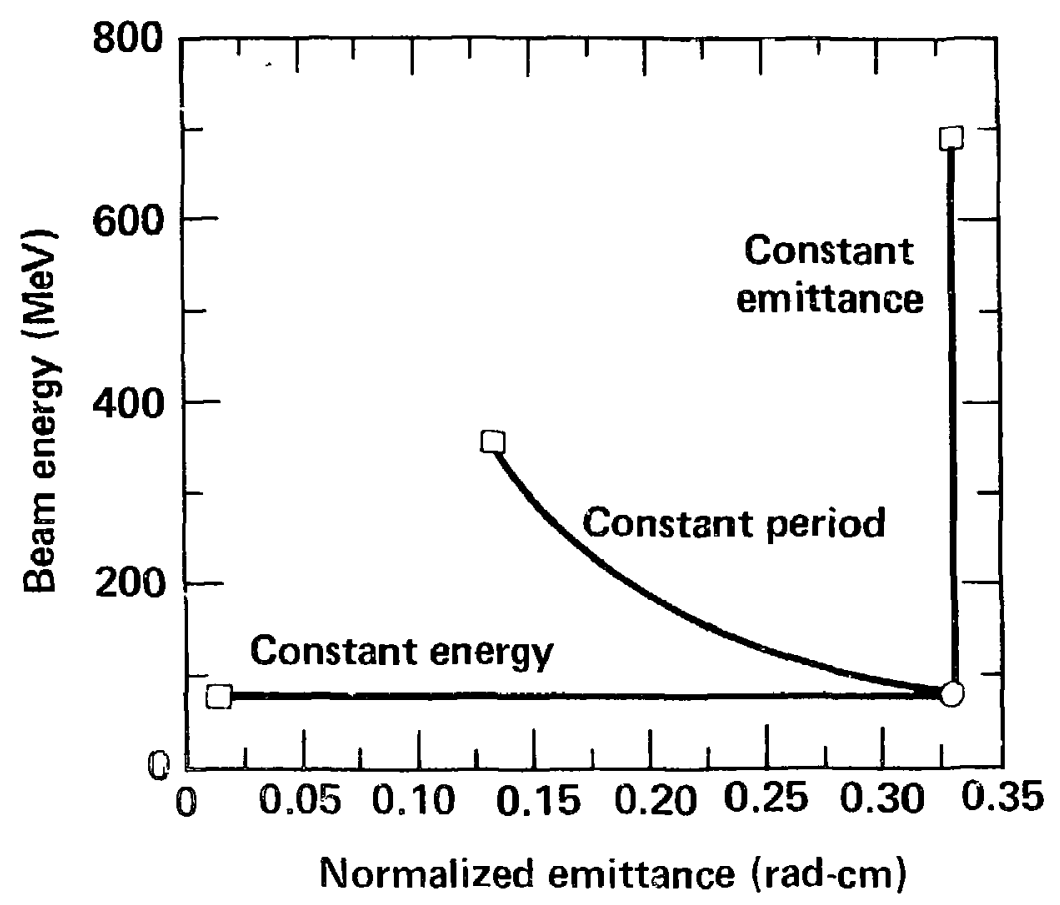


- High electric fields enhance trapping

$$
\left.\frac{\Delta \gamma_{\|}}{\gamma}\right)_{\text {trapped }} \alpha \mathrm{E}_{\mathrm{s}}^{1 / 2}
$$

- High electric fields are required to decelerate the electron beam

$$
\frac{\mathrm{d} \gamma}{\mathrm{dZ}} \alpha-\mathrm{E}_{\mathrm{s}} \approx\left(\frac{2.9 \mathrm{MeV}}{\mathrm{m}}\left(\frac{\mathrm{I}}{100 \mathrm{GW} / \mathrm{cm}^{2}}\right)^{1 / 2}\right)
$$

- High currents are required for high gains

$$
\frac{d E_{s}}{d Z} \alpha J_{\text {trapped }}
$$


- Optical guiding - how long a wiggler is acceptable

- Exponential gain - how does the FEL saturate and what instabilities occur in very high gain systems

$B$

- Beam (both laser \& electron) profiles 


\section{Summary}

- The FEL is a "simple" enough system to be effectively modeled

- Scaling must consider for 3D effects

- Scaling must consider real hardware

- Drive laser limits

- Magnetic field limit

- Mechanical tolerances

- Gptical beam train limits

- The FEL is new enough that all designs have a safety factor built in

"No cliff designs" 